\title{
Percepção da usabilidade de sutiã: uma Revisão Bibliográfica Sistematizada (RBS)
}

\author{
Perception of bra usability: a Systematic Literature Review (SLR) \\ Percepción de usabilidad del sujetador: Una revisión de la literatura sistematizada (RBS)
}

Recebido: 22/12/2021 | Revisado: 29/12/2021 | Aceito: 04/01/2022 | Publicado: 07/01/2022

\author{
Ana Caroline Marques Miranda \\ ORCID: https://orcid.org/0000-0003-3855-2575 \\ Universidade Estadual Paulista "Júlio de Mesquita Filho", Brasil \\ E-mail: ac.miranda@unesp.br \\ Luís Carlos Paschoarelli \\ ORCID: https://orcid.org/0000-0002-4685-0508 \\ Universidade Estadual Paulista "Júlio de Mesquita Filho", Brasil \\ E-mail: luis.paschoarelli@unesp.br
}

\begin{abstract}
Resumo
O desenvolvimento, produção e comercialização de sutiãs adequados às diferentes tipologias morfológicas das mulheres, têm sido um desafio não apenas do setor produtivo (o qual deveria oferecer sutiãs com usabilidade), mas também (e principalmente) do setor de Pesquisa e Desenvolvimento (P\&D) da indústria da moda, no Brasil e no Mundo. Esta situação tem refletido em frustração e desconforto por parte das usuárias deste importante componente da moda atual. O objetivo deste estudo foi explorar, analisar e discutir os artigos científicos acerca da percepção do uso de sutiã, por mulheres adultas, a partir de uma revisão bibliográfica sistematizada. O método para a elaboração da questão foi o PICO e para o checklist do processo foi o PRISMA. Foram coletados dados nas plataformas Capes, Scielo e Scopus; e identificados 136 artigos que, ao aplicar os critérios de inclusão/exclusão e de elegibilidade, resultaram em 13 artigos elegidos. Estes artigos foram analisados de forma quantitativa, com a compreensão numérica e de forma qualitativa, na qual se analisou criticamente os conteúdos abordados. Neste sentido, o presente estudo aponta que foram identificados e discutidos diferentes estudos que reforçam a hipótese relacionada aos problemas de interação produto (sutiã) e usuária (mulheres); e que ainda faltam abordagens aplicadas visando a busca de conhecimentos que demonstrem a solução destes problemas relacionados ao sutiã.
\end{abstract}

Palavras-chave: Revisão Bibliográfica Sistemática; Sutiã, Usabilidade; Percepção; Vestuário.

\begin{abstract}
The development, production and marketing of bras suitable for different morphological types of women, have been a challenge not only of the production sector (which should offer bras with usability) but also (and especially) the Research and Development (R\&D) sector of the fashion industry, in Brazil and worldwide. This situation has been reflected in frustration and discomfort by the users of this important component of the current fashion. The objective of this study was to explore, analyze and discuss the scientific articles about the perception of bra wearing, by adult women, from a systematized literature review (SLR). The method for the elaboration of the question was the PICO and the process checklist was the PRISMA. Data were collected in the platforms Capes, Scielo and Scopus; and 136 articles were identified that, when applying the inclusion/exclusion and eligibility criteria, resulted in 13 eligible articles. These articles were analyzed quantitatively, with numerical understanding, and qualitatively, in which the contents addressed were critically analyzed. In this sense, the present study points out that different studies were identified and discussed that reinforce the hypothesis related to the problems of interaction product (bra) and user (women); and that there is still a lack of applied approaches aimed at the search for knowledge that demonstrate the solution of these problems related to the bra.
\end{abstract}

Keywords: Systematic Bibliographic Review; Bra; Usability; Perception; Clothing.

\section{Resumen}

El desarrollo, la producción y la comercialización de sujetadores adecuados a los diferentes tipos morfológicos de la mujer, han sido un desafío no sólo del sector de la producción (que debe ofrecer sujetadores con usabilidad), sino también (y especialmente) del sector de Investigación y Desarrollo (I+D) de la industria de la moda, en Brasil y en el mundo. Esta situación se ha reflejado en la frustración y el incomodidad de los usuarios de este importante componente de la moda actual. El objetivo de este estudio fue explorar, analizar y discutir los artículos científicos sobre la percepción del uso del sujetador, por parte de las mujeres adultas, a partir de una revisión bibliográfica sistematizada. El método para la elaboración de la pregunta fue el PICO y para la lista de comprobación del proceso fue el PRISMA. Los datos se recogieron en las plataformas Capes, Scielo y Scopus; y se identificaron 136 artículos que, al aplicar los criterios de inclusión/exclusión y elegibilidad, dieron como resultado 13 artículos elegibles. Estos artículos se analizaron de forma cuantitativa, con comprensión numérica, y cualitativa, en la que se analizaron 
críticamente los contenidos abordados. En este sentido, el presente estudio señala que se identificaron y discutieron diferentes estudios que refuerzan la hipótesis relacionada con los problemas de interacción producto (sujetador) y usuario (mujer); y que aún faltan enfoques aplicados dirigidos a la búsqueda de conocimientos que demuestren la solución de estos problemas relacionados con el sujetador.

Palabras clave: Revisión Bibliográfica Sistemática; Sujetador; Usabilidad; Percepción; Ropa.

\section{Introdução}

O Vestuário é um artefato que está presente no cotidiano do ser humano, nas mais diferentes culturas, nos mais distintos grupos sociais e etários, sendo parte constitutiva da sociedade moderna. Embora o vestuário tenha surgido na PréHistória, as vestes íntimas foram mais fortemente adotadas no decorrer da Idade Média, após o surgimento da moda; e no Século XX, sua popularização e disseminação podem ser considerados fenômenos sociais contemporâneos (Alves \& Martins, 2018).

De fato, o que pode ser classificado como vestes íntimas é aquela que, necessariamente mantém-se em contato direto com a pele (Rosseti, 1995); e sua evolução está associada ao desdobramento da indústria têxtil e também à moda das vestimentas, especialmente como decorrência do capitalismo (Scott, 2013). A partir de então, como consequência da produção em larga escala e de avanços tecnológicos na produção de tecidos, o sutiã começou a ser popularizado, visando suprir a necessidade de um maior conforto na vida cotidiana das mulheres.

O sutiã conquistou seu próprio papel na história do vestuário, com os primeiros registros ainda na antiga Creta, mas somente em 1914 é que foi oficialmente patenteado. E, apesar dos avanços tecnológicos neste segmento do vestuário e da moda, o sutiã ainda é sinônimo de frustração entre as mulheres por diversas razões. Pode-se destacar, por exemplo: os problemas relacionados à sua dimensão, a qual pode não ser condizente com as reais variabilidades antropométricas das usuárias; os incômodos apresentados pelo emprego de materiais e/ou pelas tecnologias de produção das diferentes partes e componentes; os pontos de pressão sobre a pele, causados por partes e/ou componentes (p.e., a alça); as dificuldades de transpiração da pele da usuária, em razão do material utilizado não ser o mais adequado ao uso do artefato; entre inúmeros outros fatores.

Neste sentido, o sutiã (enquanto produto) caracteriza-se pela própria interface entre corpo e vestuário; e seu design deve estar comprometido em proporcionar saúde, bem-estar, conforto e satisfação das usuárias. Além disto, o sutiã é considerado o produto líder da linha de lingerie, ou seja, 55\% desta linha a partir de 2016 (Shahbandeh, 2021). Segundo esta autora, isto se deve ao fato de serem empregados esforços para que o sutiã se torne cada vez mais inclusivo, considerando os diferentes tipos de corpos.

Mediante o contexto apresentado, este artigo teve como objetivo explorar, analisar e discutir os estudos acerca da percepção da usabilidade dos sutiãs, por mulheres adultas, a partir de uma revisão bibliográfica sistematizada.

\section{Metodologia}

O presente estudo foi desenvolvido com base em uma Revisão Bibliográfica Sistemática - RBS, cuja estrutura metodológica está fundamentada nas do Preferred Reporting Items for Systematic Reviews and Meta-Analyses - PRISMA (Moher, et al., 2009).

A ferramenta proposta envolve o método PICO (População, Intervenção, Controle e Outcome), então empregado para a formulação da questão da pesquisa, visto que auxilia na composição dos seus respectivos componentes, de forma precisa e focada (Ferreira \& Abrahão, 2021; Santos, et al., 2007).

Neste sentido, a questão de pesquisa definida foi: Como usuárias de sutiã (P) percebem (I) os diferentes designs (C) deste vestuário e como isto interfere na usabilidade (O) do mesmo? 
A partir deste delineamento metodológico, foi possível especificar os descritores (na língua Inglesa) que seriam aplicados nas etapas seguintes, conforme apontado no Quadro 1.

Quadro 1 - Descritores (na língua Inglesa) do PICO.

\begin{tabular}{|c|l|l|}
\hline $\mathbf{P}$ & População & 'woman' or 'users' \\
\hline $\mathbf{I}$ & Intervenção & 'perception' or 'interaction' \\
\hline $\mathbf{C}$ & Controle & 'bra'or 'brassiere' or 'design of bra' \\
\hline $\mathbf{O}$ & Outcome & usability \\
\hline
\end{tabular}

Fonte: Elaborada pelos autores (2021).

A partir desta definição de descritores, os mesmos foram aplicados na busca de estudos nas bases de dados da Capes, Scielo e Scopus, as quais direcionaram inicialmente para o campo da saúde, sendo necessário então, adicionar o descritor 'Clothing' para segmentar vestuário/moda íntima. Cada plataforma apresentou um comportamento que exigiu que a busca fosse refeita com os seguintes descritores: 'Bra' OR 'Brassiere' AND 'Clothing' AND 'Usability' AND 'Interation' OR 'Perception'.

Nas plataformas Capes e Scielo, adotou-se inicialmente a filtragem pelos últimos cinco anos. Contudo os resultados não foram muito representativos, o que levou à extensão de 11 anos (período de 2010 a 2021) nas três plataformas. Em decorrência disto, os resultados mais relevantes foram com os descritores: 'Bra' AND 'Clothing' AND 'Usability' AND 'Perpection'. Este levantamento, foi realizada no período de abril e maio de 2021, e conforme o Quadro 2 abaixo, foi necessário adotar critérios para a triagem dos estudos.

Quadro 2 - Critérios de seleção.

\begin{tabular}{|c|ll|}
\hline Critérios de inclusão & $\bullet$ & Artigos originais dos últimos 11 anos \\
\hline \multirow{3}{*}{ Critério para exclusão } & $\bullet$ & Estudos duplicados, \\
& $\bullet$ & Trabalhos de conclusão de curso. \\
& $\bullet$ & Artigos incompletos \\
& $\bullet$ & Links que não acessam \\
\hline \multirow{2}{*}{ Filtros Adotados } & $\bullet$ & FILTRO 1 - Análise do título \\
& $\bullet$ & FILTRO 2 - Leitura do abstract \\
& $\bullet$ & FILTRO 3 - Leitura da Introdução \\
\hline \multirow{2}{*}{ Critérios para elegibilidade } & $\bullet$ & Estudos sobre percepção feminina sobre o próprio \\
& corpo; & \\
& $\bullet$ & Estudos diversos sobre o sutiã; \\
& $\bullet$ & Estudos sobre usabilidade no vestuário; \\
& $\bullet$ & Estudos sobre antropometria do corpo feminino; \\
& $\bullet$ & Estudos sobre desconforto no vestuário. \\
\hline
\end{tabular}

Fonte: Elaborada pelos autores (2021).

Assim chegou-se em 21 artigos, dos quais 8 foram posteriormente excluídos, pois alguns não permitiram o acesso; e outros, ao aplicar o Filtro 3, não apresentavam-se correlacionados ao assunto em questão. Assim sendo, com a aplicação dos critérios para elegibilidade, foram definidos 13 artigos finais.

Para auxiliar nos resultados, foi utilizada a ferramenta Prisma, cujo seu objetivo é auxiliar os autores, em um checklist de todo processo desenvolvido. (Galvão, et al., 2015) A Figura 1, foi elaborado um organograma com a resolução do processo. 
Figura 1 - Análise dos dados

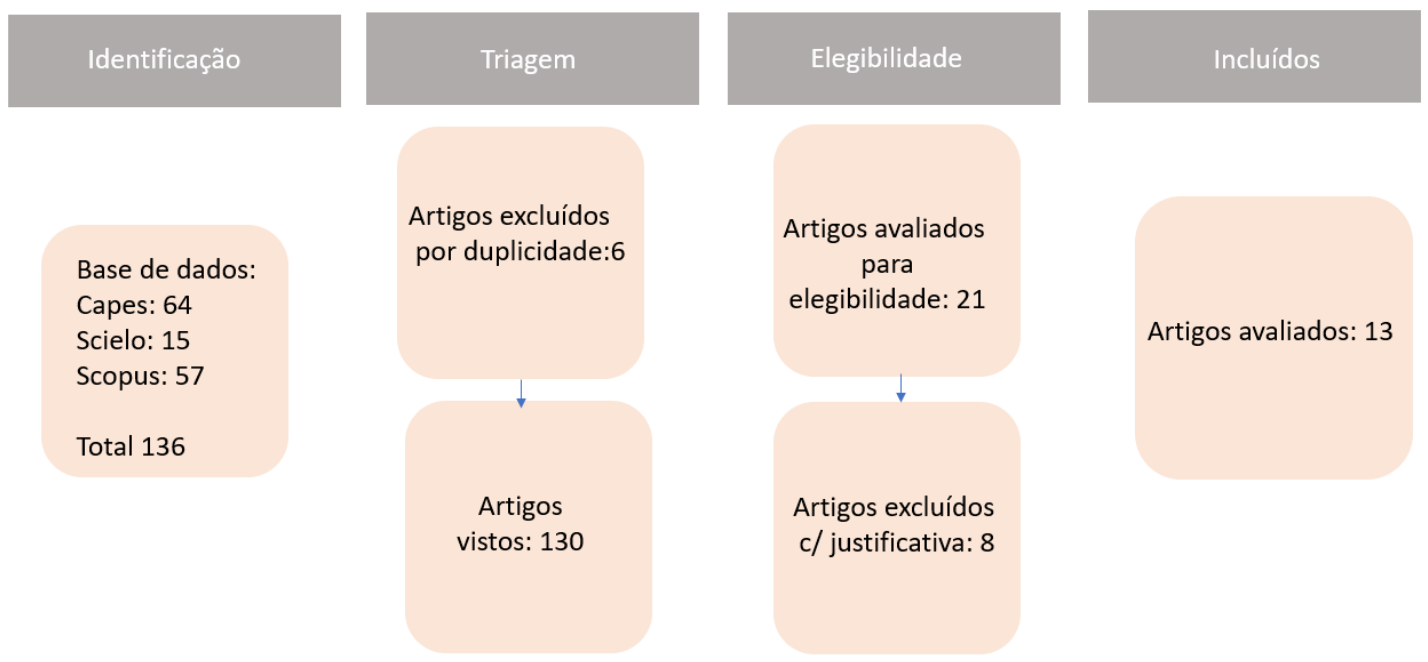

Fonte: Elaborada pelos autores (2021).

\section{Resultados}

Após análise dos artigos elegidos, as informações coletadas foram segmentadas de forma quantitativa e de forma qualitativa, na qual se analisou o conteúdo dos artigos selecionados. No Quadro 3, estão os 13 artigos selecionados, para melhor compreensão, eles foram estruturados por ano, dos mais antigos para os mais recentes.

Quadro 3 - Artigos Elegidos.

\begin{tabular}{|c|c|c|c|c|}
\hline Ano & Autores & Periódico & Título & Citações \\
\hline 2010 & $\begin{array}{l}\text { CHEN, C.M.; } \\
\text { LABAT, K.; } \\
\text { BYE, E. }\end{array}$ & Ergonomics & Physical characteristics related to bra fit & 19 \\
\hline 2011 & $\begin{array}{l}\text { CHEN, C.M.; } \\
\text { LABAT, K.; } \\
\text { BYE, E. }\end{array}$ & $\begin{array}{c}\text { International } \\
\text { Journal of } \\
\text { Consumer Studies }\end{array}$ & $\begin{array}{c}\text { Bust prominence related to bra fit } \\
\text { problems }\end{array}$ & 13 \\
\hline 2012 & $\begin{array}{l}\text { FILIPE, A.B.; } \\
\text { MONTAGNA, G.; } \\
\text { CARVALHO, C.; } \\
\text { MOREIRA, S.F. }\end{array}$ & $\begin{array}{l}\text { Book Chapter - } \\
\text { Human Factors } \\
\text { and Ergonomics } \\
\text { in Manufacturing }\end{array}$ & $\begin{array}{c}\text { Breast design: The role of ergonomic } \\
\text { underwear during the lifetime }\end{array}$ & - \\
\hline 2013 & $\begin{array}{l}\text { ALVES, R.P.; } \\
\text { MARTINS, L.B.; } \\
\text { MARTINS, S.B. }\end{array}$ & $\begin{array}{c}\text { Occupational } \\
\text { Safety and } \\
\text { Hygiene - } \\
\text { Proceedings of } \\
\text { the International } \\
\text { Symposium on } \\
\text { Occupational } \\
\text { Safety and } \\
\text { Hygiene }\end{array}$ & $\begin{array}{c}\text { Comfort underwear, their implications for } \\
\text { women's Heath in task } \\
\text { performance }\end{array}$ & 2 \\
\hline 2014 & $\begin{array}{l}\text { RISIUS, D.; } \\
\text { THELWELL, R.; } \\
\text { WAGSTAFF, C.R.D.; } \\
\text { SCURR, J. }\end{array}$ & $\begin{array}{l}\text { European Journal } \\
\text { of Ageing }\end{array}$ & $\begin{array}{c}\text { The influence of ageing on bra preferences } \\
\text { and self-perception of breasts among } \\
\text { mature women }\end{array}$ & 13 \\
\hline 2015 & $\begin{array}{l}\text { VIANNA, C.; } \\
\text { QUARESMA, M. }\end{array}$ & $\begin{array}{c}\text { Procedia } \\
\text { Manufacturing }\end{array}$ & $\begin{array}{l}\text { Ergonomic Issues Related to Clothing and } \\
\text { Body Changes of the New Elderly Women }\end{array}$ & 3 \\
\hline 2015 & $\begin{array}{l}\text { BURNETT, E.; } \\
\text { WHITE, J.; } \\
\text { SCURR, J. }\end{array}$ & $\begin{array}{l}\text { Journal of } \\
\text { Physical Activity } \\
\text { and Health }\end{array}$ & $\begin{array}{l}\text { The influence of the breast on physical } \\
\text { activity participation in females }\end{array}$ & 28 \\
\hline 2016 & LIU, Y; & The Journal of & Study of optimum parameters for Chinese & 5 \\
\hline
\end{tabular}




\begin{tabular}{|c|c|c|c|c|}
\hline & $\begin{array}{l}\text { WANG, J.; } \\
\text { ISTOOK, C.L. }\end{array}$ & $\begin{array}{c}\text { The Textile } \\
\text { Institute }\end{array}$ & $\begin{array}{c}\text { female underwire bra size system by } 3 D \\
\text { virtual anthropometric measurement }\end{array}$ & \\
\hline 2017 & $\begin{array}{l}\text { ZHU, X.; } \\
\text { TAMURA, T.; } \\
\text { KOSHIBA, T. }\end{array}$ & $\begin{array}{l}\text { Japan Research } \\
\text { Association for } \\
\text { Textile End-Uses }\end{array}$ & $\begin{array}{c}\text { An experimental study on the vibration } \\
\text { control of brassieres designed for different } \\
\text { breast sizes and shapes }\end{array}$ & 1 \\
\hline 2017 & $\begin{array}{l}\text { SATSUMOTO, Y.A.; } \\
\text { MARUTA, N.B.; } \\
\text { SAITO, H.C; } \\
\text { MOROOKA, H.D. }\end{array}$ & Ergonomics & $\begin{array}{l}\text { Effect of age, body features and type of } \\
\text { brassiere on the comfort in movement }\end{array}$ & 1 \\
\hline 2018 & $\begin{array}{l}\text { BISHOP, K.; } \\
\text { GRUYS, K.; } \\
\text { EVANS, M. }\end{array}$ & $\begin{array}{l}\text { Gender and } \\
\text { Society }\end{array}$ & $\begin{array}{c}\text { Sized Out: Women, Clothing Size, and } \\
\text { Inequality }\end{array}$ & 8 \\
\hline 2020 & $\begin{array}{l}\text { MOTA, V.E.C.; } \\
\text { HAIKAL, D. S.; } \\
\text { MAGALHÃES, T.A.; } \\
\text { SILVA, N.S.S.; } \\
\text { SILVA, R.R.S. }\end{array}$ & $\begin{array}{l}\text { Revista de } \\
\text { Nutrição }\end{array}$ & $\begin{array}{l}\text { Dissatisfaction with body image and } \\
\text { associated factors in adult women }\end{array}$ & - \\
\hline 2021 & $\begin{array}{l}\text { FANG, F.; } \\
\text { HU, C.; } \\
\text { YAN, T.T. }\end{array}$ & $\begin{array}{c}\text { Article } \\
\text { Source type } \\
\text { International } \\
\text { Journal of } \\
\text { Clothing Science } \\
\text { and Technology }\end{array}$ & $\begin{array}{l}\text { Research on bra component design and } \\
\text { perceptual image prediction }\end{array}$ & - \\
\hline
\end{tabular}

Fonte: Elaborada pelos autores (2021).

\subsection{Análise quantitativa}

$\mathrm{Na}$ análise dos resultados quantitativos, ficou evidenciado que os estudos se originam em 6 (seis) diferentes países, na qual a China se destaca com 4 estudos a respeito do sutiã, conforme indicado na Figura 2.

Figura 2 - Relação dos artigos por país.

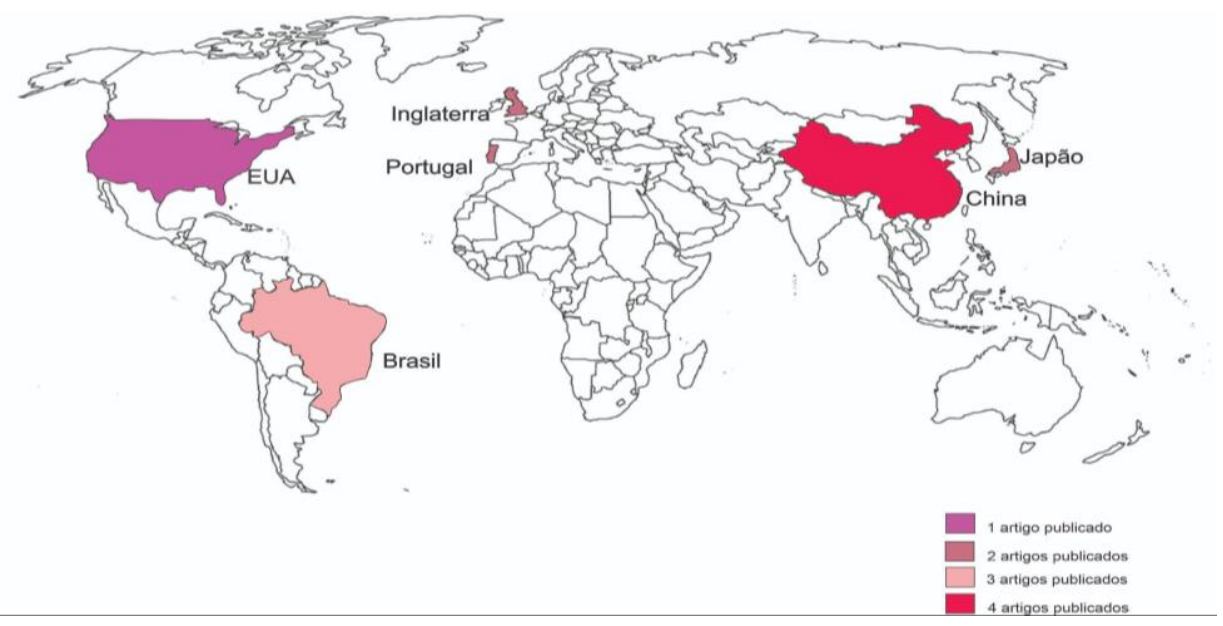

Fonte: Elaborada pelos autores (2021).

Outro aspecto notado foi a quantidade de citações desses estudos, no qual se destaca The influence of the breast on physical activity participation in females (Burnett, et al., 2015) com 28 citações, seguido do Physical characteristics related to bra fit (Chen, et al., 2010) com 19 citações; e com 13 citações têm-se Bust prominence related to bra fit problems (Chen, et al., 2011) e The influence of ageing on bra preferences and self-perception of breasts among mature women (Risius, et al., 2014). Isto demonstra que, apesar do número discreto de artigos destacados nesta RBS, o número de citações de alguns deles pode ser considerado representativo. 


\subsection{Análise qualitativa}

Com relação a análise qualitativa, observou-se uma segmentação na abordagem dos estudos, assim para facilitar a compreensão destes conteúdos, os artigos foram fracionados dentro das temáticas identificadas, conforme apontado no Quadro 4.

Quadro 4 - Segmentação dos estudos.

\begin{tabular}{|c|c|}
\hline \multirow{3}{*}{$\begin{array}{l}\text { ESTUDOS SOBRE IMPACTO } \\
\text { EM IDOSAS }\end{array}$} & GRUPOS DE ESTUDO \\
\hline & $\begin{array}{l}\text { Ergonomic Issues Related to Clothing and Body Changes of the New } \\
\text { Elderly Women (2015) }\end{array}$ \\
\hline & $\begin{array}{l}\text { The influence of ageing on bra preferences and self-perception of breasts } \\
\text { among mature women (2014) }\end{array}$ \\
\hline \multirow{3}{*}{$\begin{array}{l}\text { ESTUDOS SOBRE } \\
\text { PERCEPÇÕES DIVERSAS } \\
\text { SOBRE O SUTIÃ } \\
\end{array}$} & Research on bra component design and perceptual image prediction (2021) \\
\hline & $\begin{array}{l}\text { Comfort underwear, their implications for women's health in task } \\
\text { performance (2013) }\end{array}$ \\
\hline & Bust prominence related to bra fit problems (2011) \\
\hline \multirow{2}{*}{$\begin{array}{c}\text { ESTUDOS DE } \\
\text { DIMENSIONAMENTO COM } \\
\text { SCANNER 3D } \\
\end{array}$} & $\begin{array}{l}\text { Study of optimum parameters for Chinese female underwire bra size } \\
\text { system by 3D virtual anthropometric measurement (2016) }\end{array}$ \\
\hline & Physical characteristics related to bra fit (2010) \\
\hline $\begin{array}{c}\text { ESTUDOS SOBRE } \\
\text { INSATISFAÇÃO COM O } \\
\text { PRÓPRIO CORPO } \\
\end{array}$ & $\begin{array}{l}\text { Dissatisfaction with body image and associated factors in adult women } \\
(2020)\end{array}$ \\
\hline \multirow{2}{*}{$\begin{array}{l}\text { ESTUDOS SOBRE A MAMA } \\
\text { FEMININA }\end{array}$} & $\begin{array}{l}\text { The influence of the breast on physical activity participation in females } \\
\text { (2015) }\end{array}$ \\
\hline & Breast design: The role of ergonomic underwear during lifetime (2012) \\
\hline
\end{tabular}

Fonte: Elaborada pelos autores (2021).

\section{Discussão}

O primeiro grupo identificado foi a respeito do uso do sutiã e a consequência deste artefato com as novas idosas, como o estudo brasileiro, Ergonomic Issues Related to Clothing and Body Changes of the New Elderly Women, das autoras Vianna e Quaresma (2015), no qual o foco deste estudo está nas mudanças ocorridas no corpo feminino, conforme os estágios da vida e as dificuldades que há no vestuário durantes esta transição. Através de um grupo em foco, as autoras questionaram a respeito da preferência das roupas. Ficou relatado a importância que o designer deve ter na escolha do material para a construção de uma peça e como as novas idosas, que possuem comportamento social mais ativo, sentem necessidade de roupas elaboradas a partir do conforto. O principal resultado a defasagem no mercado do vestuário para este público, principalmente peças com tecidos naturais. Já no estudo inglês, os autores Risius, et al., (2014), The influence of ageing on bra preferences and self-perception of breasts among mature women, analisaram um grupo de mulheres de 45 a 65 anos, como o envelhecimento influencia a percepção das mulheres sobre seus seios e quais são as preferências para sutiãs. Ficou detectado que a principal importância para os participantes foram: conforto, ajuste adequado, boa aparência sob as roupas, elevação dos seios. Além de influenciar na feminilidade, também está ligando a sexualidade, o sutiã se torna uma expressão desta identidade. $\mathrm{O}$ artigo ainda frisa para os fabricantes se atentarem em combinações estética (fatores externos) e funcional (fatores internos).

Já no segundo grupo, as temáticas são trabalhadas a partir da percepção pessoal feminina, no estudo chinês Research on bra component design and perceptual image prediction, realizado pelos autores Fang, et al., (2021) com uma amostragem 
feminina de 18 a 27 anos, sobre a percepção que as usuárias têm a respeito dos componentes do sutiã e qual o efeito que possuem sobre elas, para esta análise foi escolhido o método de psicologia cognitiva, seguido de dois experimentos.

No experimento, ficou identificado que as usuárias, chinesas, mostraram que estes componentes têm impacto na percepção visual e também ficou identificado 5 componentes que mais afetam: copa, altura frontal central, largura do tronco, largura lateral e formato traseiro central. Os autores ainda complementam que os resultados desta pesquisa, pode auxiliar as empresas a determinar um sistema de forma assertiva para minimizar os problemas gerados pelo ajuste. No estudo brasileiro Comfort underwear, their implications for women's health in task performance, das autoras Alves, et al., (2013) o conforto e usabilidade da roupa intima (calcinha e sutiã) utilizada no dia a dia, foram analisados, assim como suas implicações na saúde. As vestes intimas analisadas, mostraram tanto desconforto, quanto limitações em seu uso, além de consequências negativas à saúde feminina, na execução de trabalhos. As autoras ainda frisam a necessidade de uma harmonia entre materiais, modelos, acabamento para que a saúde e bem-estar sejam preservados. No estudo chinês, Bust prominence related to bra fit problems, dos autores Chen, et al., (2011) o foco está em analisar como o volume do seio impactam no uso do sutiã no dia a dia. A pesquisa foi desenvolvida com uma amostragem feminina de 103 mulheres jovens, universitárias, de 18 a 25 anos, que responderam a um questionário, para detectar as percepções sobre o sutiã no ambiente escolar e de trabalho. O que ficou destacado foi que a falta de sustentação é o problema mais grave, mulheres com mais volumes ainda detectaram falta de ajuste na copa e alças, também sem queixaram de fechos desconfortáveis.

Neste grupo com o uso de scanner 3D, temos o estudo chinês, Study of optimum parameters for Chinese female underwire bra size system by $3 D$ virtual anthropometric measurement, dos autores Liu, et al., (2016), o foco está em encontrar parâmetros para o desenvolvimento do sutiã ideal, através das medidas da mama feminina, a pesquisa foi realizada com 275 estudantes chinesas, de 18 a 35 anos. A análise dos dados foi realizada juntamente com método de peso da entropia e os métodos da Análise de Componentes Principais (PCA). Para o estudo, as mulheres, foram orientadas a vestir uma calcinha justa, sem sutiã ou outra cobertura na parte superior do corpo e uma touca para cobrir os cabelos; permaneceram eretas durante a digitalização do scanner no corpo, com saltos variados de 10 a $15 \mathrm{~cm}$ e os braços mantidos separados dos lados do torso. Já neste outro estudo chinês, Physical characteristics related to bra fit, os autores Chen, et al., (2010) investigam a relação das características físicas com os problemas de ajuste, com o método Scanner 3D, Scanner VITUS Smart (Human Solutions $\mathrm{GmbH}$, Kaiserslautern, Alemanha), extraem dados da parte superior de uma amostra de 103 mulheres, de 18 a 25 anos. Diferente do outro estudo, neste as mulheres, usam o sutiã do dia a dia, e o scanner é utilizada nelas com o sutiã. A investigação de ajuste é feita através de uma análise estatística. Os dados extraídos foram utilizados para categorizar as características físicas e a somatória destas categorizações, juntamente com os problemas de caimento foram então examinados. Concluindo que o formato do corpo é um fator importante que impacta diretamente no ajuste, o estudo também coloca como sugestão para futuras pesquisa, a questão do volume da mama.

No quesito de insatisfação pessoal, o estudo brasileiro Dissatisfaction with body image and associated factors in adult women, dos autores Mota, et al., (2020) que abordam a percepção que as mulheres possuem sobre o próprio corpo, foi com uma amostragem de 633 mulheres, todas professoras do primário, de diversas idades. Foi feito um levantamento, através de questionário, sobre peso, altura (para calcular IMC) e como classificavam a saúde do próprio corpo. O estudo identificou que quase $50 \%$ estão insatisfeitas com o corpo, principalmente mulheres mais velhas, acima do peso e que têm filhos. Mulheres com quadro depressivo apresentam maior insatisfação, também se concluiu que a mídia tem um impacto grande na forma como a mulher enxerga o próprio corpo.

Por fim, os estudos acerca da mama feminina, trazem perspectivas diferentes, no estudo português, Breast design: The role of ergonomic underwear during lifetime, os autores Filipe, et al., (2012) desenvolveram um projeto de criação de sutiã com o apoio de uma empresa do setor, e ao estudar a peças identificaram os diferentes estágios que a mama feminina, passa ao 
longo da vida, desde que se desenvolve (na adolescência até no envelhecimento). Ficou claro que os diferentes formatos da mama, a torna tão singular e o quanto isto dificulta o design de peça baseado no conforto. Os autores ainda concluem que a lingerie está ligada ao fator emotivo, pois impacta na forma se sentem com uma peça que veste bem. Já o estudo inglês, The influence of the breast on physical activity participation in females, os autores Burnett, et. al, (2015) relatam a dificuldade da mulher, na escolha de um sutiã para atividade física. Investigam se a mama tem influência de alguma maneira, na escolha da atividade física e a consequência do sutiã na mesma. O que se destaca neste estudo foi como os níveis de atividades físicas repercutem: são menores naquelas que sentem dores no seio. Entre os pontos levantados foi a dificuldade de não encontrar sutiã esportivo adequado e quando encontram, se incomodam com a aparência que o seio fica nele. A mama é uma barreira que a mulher tem ao se exercitar e isto impacta diretamente na auto estima feminina, a consequência é que muitas mulheres não se exercitam por vergonha.

Infelizmente no decorrer desta investigação, houve 3 estudos selecionados pelos critérios de elegibilidade, que não estavam disponíveis e mesmo assim houve a tentativa de contato com os autores, no entanto, sem sucesso: o estudo americano Sized Out: Women, Clothing Size, and Inequality, dos autores Bishop, et al., (2018), o estudo japonês An experimental study on the vibration control of brassieres designed for different breast sizes and shapes, dos autores Zhu, et al., (2017). Já o estudo Effect of age, body features and type of brassiere on the comfort in movement, os autores Satsumoto, et al., (2016), retornaram com o material, no entanto, como está escrito em japonês não atende ao critério de inclusão selecionado inicialmente.

\section{Considerações Finais}

O desenvolvimento de um sutiã pode ser desafiador para a indústria, visto que diversos são os pontos que envolvem uma criação bem elaborada e impactam na usabilidade. Neste caso, podemos destacar, a diversidade morfológica do corpo feminino e incluem-se aqui "os formatos" das mamas; o aspecto emocional das usuárias; e até mesmo a influência da mídia.

O presente estudo teve como propósito apresentar uma Revisão Bibliográfica Sistematizada afim de esclarecer como usuárias deste artefato/produto percebem seus diferentes designs; e como isto interfere na usabilidade do mesmo.

Os resultados apontam que as pesquisas encontradas e analisadas ajudam a esclarecer o propósito do presente estudo, contribuindo com abordagens expressivas para se compreender quais fatores devem ser considerados quando se busca a usabilidade dos sutiãs. Portanto, o conhecimento explorado pode ser um fator expressivo para o setor produtivo, sendo que alguns estudos já procuram buscar o emprego das novas tecnologias, com destaque para os problemas relacionados à adequação dimensional do artefato/produto em questão, sendo ainda essencial o desdobramento de novos estudos com outros grupos femininos para explorar, comparar, a fim de verificar se as dificuldades são as mesmas ou não.

Em suma, há diversas lacunas a respeito do artefato/produto em questão, os quais poderão ser esclarecidos com uma abordagem focada na percepção efetiva das usuárias sobre diferentes fatores. O Design Ergonômico poderá ser uma alternativa metodológica para esta compreensão.

\section{Agradecimentos}

O presente estudo foi desenvolvido com apoio da CAPES - Coordenação de Aperfeiçoamento de Pessoal de Nível Superior (Processo 88887.636925/2021-00).

\section{Referências}

Alves, R. P., Martins, L. B., \& Martins, S. B. (2013) Comfort underwear, their implications for women's Heath in task performance. Occupational Safety and Hygiene - Proceedings of the International Symposium on Occupational Safety and Hygiene. 27-302. 10.1201/b14391-7 
Alves, R. P., \& Martins, L. B. (2018) O sutiã e Seus precursores: uma análise estrutural e diacrônica. ModaPalavra - Estudos de Tendências e Branding de Moda, 11, 459-482. https://periodicos.udesc.br/index.php/modapalavra/article/view/12259/8612.

Bishop, K., Gruys, K., \& Evans, M. (2018) Sized Out: Women, Clothing Size, and Inequality. Gender and Society. 32 , $180-203$. https://doi.org/10.1177/0891243218756010

Burnett, E., White, J., \& Scurr, J. (2015) The Influence of the Breast on Physical Activity Participation in Females. J Phys Act Health. 10.1123/jpah.20130236

Chen, C. M., Labat, K., \& Bye E. (2010) Physical characteristics related to bra fit. Ergonomics. V. 53, Issue 4, P. 514-524. 10.1080/00140130903490684.

Chen, C. M., Labat, K., \& Bye E. (2011) Bust prominence related to bra fit problems. International Journal of Consumer Studies. v.35, Issue6, p. 695-701. https://doi.org/10.1111/j.1470-6431.2010.00984.x

Fang, F., Hu, C., \& Yan T. T. (2021) Research on bra component design and perceptual image prediction. International Journal of Clothing Science and Technology. 10.1108/IJCST-12-2019-0186

Ferreira, A. S., \& Abrahão, A. L. (2020) Nursing care management in the Family Health Strategy: systematic review. Research, Society and Development, 9(5). https://doi.org/10.33448/rsd-v9i5.3087

Filipe, A. B., Montagna, G., Carvalho, C., \& Silva, F. M. (2012) Breast design: The role of ergonomic underwear during lifetime. Advances in Usability Evaluation, $\quad 617 \quad-\quad 626 . \quad \mathrm{https} / / / \mathrm{www}$. scopus.com/record/display.uri?eid=2-s2.0-85055928911\&origin=resultslist\&sort=plf$\mathrm{f} \& \mathrm{src}=\mathrm{s} \& n l o=\& \mathrm{nlr}=\& \mathrm{nls}=\& \mathrm{sid}=\mathrm{f} 66 \mathrm{e} 70 \mathrm{c} 9 \mathrm{~b} 21 \mathrm{a} 3 \mathrm{a} 3 \mathrm{e} 0 \mathrm{~d} 12 \mathrm{a} 5605 \mathrm{~b} 2 \mathrm{c} 1 \mathrm{bc} 8 \& \mathrm{sot}=\mathrm{b} \& \mathrm{sdt}=\mathrm{cl} \& \mathrm{cluster}=$ scosubtype $\% 2 \mathrm{c} \% 22 \mathrm{ch} \% 22 \% 2 \mathrm{ct} \& \mathrm{sl}=48 \& \mathrm{~s}=\% 28 \mathrm{TITLE}-$

ABS-KEY\%28bra\%29+AND+TITLE-ABS-KEY\%28clothing\% 29\%29\&relpos=2\&citeCnt=0\&searchTerm=

Galvão, T. F., Pansani, T. S. A., \& Harrad, D. (2015) Principais itens para relatar revisões sistemáticas e meta-análises: a recomendação prisma. Epidemiologia e serviços de saúde . https://doi.org/10.5123/S1679-49742015000200017

Liu, Y., Wang, J., \& Istook, C. L. Study of optimum parameters for Chinese female underwire bra size system by 3D virtual anthropometric measurement. (2016) The Journal of The Textile Institute. v. 108. 877-882. 10.1080/00405000.2016.1195954

Mota, V. E. C, Haikal, D. S., Magalhães, T. A., Silva N.S. S., \& Silva, R. R. V. Dissatisfaction with body image and associated factors in adult women. Revista de Nutrição v. 33. https://doi.org/10.1590/1678-9865202033e190185

Moher D., Liberati A., Tetzlaff J., \& Altman D. G. (2009) Preferred Reporting Items for Systematic Reviews and Meta-Analyses: The PRISMA Statement. Plos Medicine. https://doi.org/10.1371/journal.pmed.1000097

Risius, D., Thelwell, R., Wagstaff, C. R. D., \& Scurr J. (2014) The influence of ageing on bra preferences and self-perception of breasts among mature women. European Journal of Ageing. v. 11, 233-240. 10.1007/s10433-014-0310-3

Rosseti, A. (1995) Roupas íntimas: o tecido da sedução. Martins Fontes.

Santos, C. M. C, Pimenta, A. M., \& Nobre, M. R. C. (2007) A estratégia PICO para a construção da pergunta de pesquisa e busca de evidências. Rev. LatinoAm. Enfermagem, V.15. https://doi.org/10.1590/S0104-11692007000300023

Satsumoto, Y., Maruta N., Saito, H., \& Morooka, H. (2017) Effect of age, body features and type of brassiere on the comfort in movement. Journal of the Japan Research Association for Textile End-Uses. 58, 80-89. https://www.scopus.com/record/display.uri?eid=2-s2.085018648381\&origin=resultslist\&sort=plf-f\&src=s\&nlo=\&nlr=\&nls=\&sid=83c3e01910c15e62893dbdcd46354578\&sot=b\&sdt=b\&sl=48\&s=\%28TITLEABS-KEY\%28bra\%29+AND+TITLE-ABS-KEY\%28clothing\%29\%29\&relpos=53\&citeCnt=0\&searchTerm=

Scott, L. (2013) Lingerie: da antiguidade à cultura pop. (ed.) Barueri: Manole.

Shahbandeh, M. (2021) Women's lingerie market value worldwide from 2018 to 2027 (in billion U.S. dollars) https://www.statista.com/statistics/720288/lingerie-retail-market-value/.

Vianna C., \& Quaresma M. (2015) Ergonomic Issues Related to Clothing and Body Changes of the New Elderly Women. Procedia Manufacturing. 3, 5755 5760. https://doi.org/10.1016/j.promfg.2015.07.819

Zhu, X, Tamura, T., \& Koshiba, T. (2017) An experimental study on the vibration control of brassieres designed for different breast sizes and shapes. Journal of the Japan Research Association for Textile End-Uses. 58, $42-52 . \quad$ https://www.scopus.com/record/display.uri?eid=2-s2.0$85026817923 \&$ origin $=$ resultslist $\&$ sort $=$ plf $-\& s r c=s \& n l o=\& n l r=\& n l s=\& s i d=83 c 3 e 01910 \mathrm{c} 15 \mathrm{e} 62893 \mathrm{dbdcd} 46354578 \&$ sot $=\mathrm{b} \& \mathrm{sdt}=\mathrm{b} \& \mathrm{sl}=48 \& \mathrm{~s}=\% 28 \mathrm{TITLE}-$

ABS-KEY\%28bra\%29+AND+TITLE-ABS-KEY\%28clothing\%29\%29\&relpos=47\&citeCnt=1\&searchTerm 